\title{
Study on the Effect of Time Delay on the Performance of Distributed Power Grids with Networked Cooperative Control
}

\author{
Junqi Liu ${ }^{\star *}$ Azwirman Gusrialdi* Dragan Obradovic ${ }^{* *}$ \\ Sandra Hirche* \\ ${ }^{*}$ Institute of Automatic Control Engineering, Technische Universität \\ München, D-80290 Munich, Germany (junqi.liu@mytum.de, \\ iman@lsr.ei.tum.de, hirche@tum.de) \\ ** Corporate Technology, Information \& Communications, Siemens \\ AG, Munich, Germany, (dragan.obradovic@siemens.com)
}

\begin{abstract}
Future Distributed Power Grid (DPG) control systems may strongly benefit from the introduction of a communication network enabling cooperation between distributed generators. However, this typically comes at the cost that network induced time delay deteriorates control performance and possibly destabilizes the overall system. In this paper we study the effect of the time delay on the performance of a DPG with networked cooperative controllers exchanging state information of generators via a communication network and analyze up to which time delay such a communication network is still beneficial for the overall control performance. Here the delay is assumed to be constant and identical for all links. Standard Linear Quadratic Regulators (LQR) are designed together with communication topology, but without explicitly considering the time delay. We compare the Linear Quadratic (LQ) cost in infinite horizon as a measure of the performance of two cases: the networked cooperative controller with global information and time delay vs. the controller with only local information. It is observed that there exists a performance guaranteed time delay bound where the cost with the cooperative controller is smaller than without information exchange, i.e. it is still beneficial to introduce a communication network. By means of a Linear Matrix Inequality (LMI) problem based on first order Pade approximation for time delay this performance guaranteed time delay bound is approximated in a systematic way. A numerical example is given to illustrate the result.
\end{abstract}

\section{INTRODUCTION}

With the ongoing introduction of novel renewable energy resources power generation units start shifting from traditional centralized, large scaled power plants to distributed and small scaled power generators. A power grid with distributed power generators, a so-called Distributed Power Grids (DGP), is more energy-efficient and produces less environmental damage.

A lot of research on the control of DPG has been conducted by considering its stability, grid connection, voltage control and power flow. Moreover, different control methods, e.g. PI control (Marwali et al. [2004]), distributed Model Predictive Control (Venkat et al. [2006]) as well as multiagent systems models (Negenborn [2007]) have been proposed.

In general, the power grid can be considered as a large scale dynamical system with interconnections due to naturally physically coupled generators. Decentralized control is a well-known area that is devoted to analyze such systems and design local control laws that use only local subsystem information in order to stabilize the overall system. Bakule

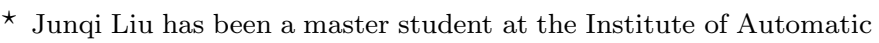
Control Engineering, Technische Universität München.
}

[2008] gives an overview on the past and present results in this area.

In future DPG, it appears promising to introduce a communication network in order to provide local controllers with non-local information allowing the cooperation between distributed generators. The resulting system contains a mixture of physical and communication interconnections between the subsystems. In addition to local information of the controlled generator each local controller may also use the information transmitted from other generators. Clearly, with instantaneous global information available to every subsystem controller in general we expect a better overall system performance compared to the decentralized approach.

However, a communication network introduces time delay which is known to deteriorate control performance and possibly destabilize the overall system. Richard [2003] gives an overview of some recent research and stabilization problems in time delay systems. In Hassan et al. [2007] decentralized optimal control laws are constructed to stabilize discrete systems with system constraints and delays with an iterative multilevel algorithm but no cooperation between subsystems. In Rotkowitz et al. [2004] the problem of decentralized control for linear interconnected system with constraints on communication topology is 
addressed. It is shown that distributed control with time delays may be reduced to a convex optimization problem when the controllers can transmit information faster than the dynamics propagate between subsystems.

In this paper we address the following practically relevant question: given a pre-designed LQR controller without consideration of time delay, what is the effect of time delay to the system performance? The main contribution of this paper is to analyze, up to which time delay value a communication network is still beneficial in terms of a LQ performance measure. The DPG dynamics are approximated by a Linear Time-Invariant (LTI) system with interconnections due to the physical couplings between the generators. Standard Linear Quadratic Regulators (LQR) are designed under the assumption of a given communication topology, but without explicitly considering the time delay. Our main goal is to study the effect of time-delay on LQ performance in such a setup, i.e. we are not implementing delay compensation methods such as e.g. in Zhang et al. [2001] . The time delay is assumed to be identical and constant for all communication links. We also assume no propagation delay exists in the physical interconnections between generators, i.e. the dynamics propagate occurs instantaneously which is different to Rotkowitz et al. [2004].

We compare the control performance of two cases: the networked cooperative control scheme with information exchange over the communication network and time delays vs. the controller with only local information and no time delay. In order to study the effect of time delay, unlike the widely applied systems decay rate in the case of exponential stabilization of time delay systems (e.g. Liu [2003]), here as a measure of performance the Linear Quadratic (LQ) cost is considered and optimal control laws for infinite horizon are implemented. It is observed that there exists a performance guaranteed time delay bound where the cost of the cooperative case is smaller than the case without information exchange, i.e. it is beneficial to introduce communication networks. This performance guaranteed time delay bound is approximated by means of Linear Matrix Inequalities (LMIs) and first order Pade approximation.

The remainder of the paper is organized as follows. In section 2 the model of the simplified DPG is introduced. In section 3 the networked cooperative and controller without information exchange are defined. The networked cooperative control with time delay is also discussed. The approximation of performance guaranteed time delay bound based on Pade approximation is introduced in section 4 . The numerical evaluation of the control performance is shown in section 5 and the conclusion is given in section 6.

\section{MODEL OF DISTRIBUTED POWER GRID}

The model of the DPG which may represent a simplified microgrid is shown in Figure 1. The DPG consists of $N$ spatially distributed power generators $G_{1} \ldots G_{N}$ supporting a constant load $R$. We assume here the generators have linear characteristics. This constant load represents the average consumption of several households in a real power grid. The generators are connected to the load by transmission lines represented by resistances $R_{1} \ldots R_{N}$. Each power

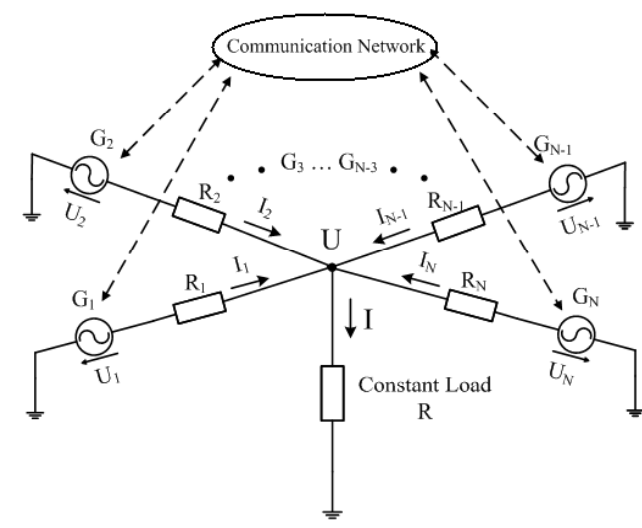

Fig. 1. Model of DPG with linear electrical DC generators

generator has a locally implemented controller and could communicate with others via the communication network.

For each individual power generator, linear electrical DC power generators model is considered. The dynamics of a linear power generator $G_{i}$ are modeled as

$$
\begin{aligned}
U_{i}(t) & =k_{i} \omega_{i}(t), \quad M_{e l, i}(t)=k_{i} I_{i}(t), \\
J_{i} \dot{\omega}_{i}(t) & =M_{m e c h, i}(t)-M_{e l, i}(t), \\
\dot{M}_{m e c h, i}(t) & =-\frac{1}{\beta_{i}} M_{m e c h, i}(t)+\frac{1}{\beta_{i}} u_{i}(t),
\end{aligned}
$$

with

- $U_{i}(t)-$ Induced voltage $[\mathrm{V}]$

- $I_{i}(t)$ - Induced current [A]

- $\omega_{i}(t)$ - Angular frequency of the rotor $[\mathrm{rad} / \mathrm{s}$ ]

- $k_{i}-$ Frequency dependency factor of the induced voltages $[\mathrm{V} \cdot \mathrm{s} / \mathrm{rad}]$

- $J_{i}$ - Moment of inertia $\left[\mathrm{kg} \cdot \mathrm{m}^{2} / \mathrm{s}^{2}\right]$

- $M_{e l, i}(t)$ - Electromagnetic torque [N $\left.\cdot \mathrm{m}\right]$

- $M_{m e c h, i}(t)$ - Mechanical torque of the rotor $[\mathrm{N} \cdot \mathrm{m}]$

- $\beta_{i}-\mathrm{A}$ time constant of mechanical torque [s]

- $u_{i}(t)-$ Driving force for the torque $[\mathrm{N} \cdot \mathrm{m}]$.

The state vector of a single generator $G_{i}$ is defined as $\mathbf{x}_{i}(t)=\left[\omega_{i}(t) M_{\text {mech }, i}(t)\right]^{T}$ and the control input is the driving force for the torque $u_{i}(t)$.

Due to Kirchhoff's law, the voltages and currents of all generators are coupled with each other. This results in that the rotor dynamics of all generators are also interconnected. The dynamics of a single generator $G_{i}$ are then given by

$$
\dot{\mathbf{x}}_{i}(t)=\mathbf{A}_{i, i} \mathbf{x}_{i}(t)+\mathbf{b}_{i} \mathbf{u}_{i}(t)+\sum_{j=1, j \neq i}^{N} \mathbf{A}_{i, j} \mathbf{x}_{j}(t),
$$

with

$$
\mathbf{A}_{i, i}=\left[\begin{array}{cc}
-\frac{k_{i}^{2} r_{i, i}}{J_{i}} & \frac{1}{J_{i}} \\
0 & -\frac{1}{\beta_{i}}
\end{array}\right] ; \quad \mathbf{A}_{i, j}=\left[\begin{array}{cc}
-\frac{k_{i} k_{j} r_{i, j}}{J_{i}} & 0 \\
0 & 0
\end{array}\right] ; \quad \mathbf{b}_{i}=\left[\begin{array}{c}
0 \\
\frac{1}{\beta_{i}}
\end{array}\right],
$$

where $r_{i, i}, r_{i, j}$ are the elements of the inverse of the resistance matrix $\mathbf{R}$ of transmission lines given by 


$$
\mathbf{R}=\left[\begin{array}{cccc}
R_{1}+R & R & \cdots & R \\
R & R_{2}+R & \ddots & \vdots \\
\vdots & \ddots & \ddots & R \\
R & \cdots & R & R_{N}+R
\end{array}\right] .
$$

Note that $\mathbf{R}$ is symmetric i.e. $r_{i, j}=r_{j, i}$. Due to space limitation we do not show its derivation here. The term $\sum_{j=1, j \neq i}^{N} \mathbf{A}_{i, j} \mathbf{x}_{j}$ in (1) represents the physical interconnections between the generators influenced by the moment of inertia $J_{i}$ and the frequency dependency factors $k_{i}, k_{j}$.

The state space representation of the DPG in Figure 1 including all generators is an LTI system with interconnections given by

with

$$
\dot{\mathbf{x}}(t)=\mathbf{A x}(t)+\mathbf{B u}(t)
$$

$$
\mathbf{A}=\left[\begin{array}{cccc}
\mathbf{A}_{1,1} & \mathbf{A}_{1,2} & \cdots & \mathbf{A}_{1, N} \\
\mathbf{A}_{2,1} & \mathbf{A}_{2,2} & \cdots & \mathbf{A}_{2, N} \\
\vdots & \vdots & \ddots & \vdots \\
\mathbf{A}_{N, 1} & \mathbf{A}_{N, 2} & \cdots & \mathbf{A}_{N, N}
\end{array}\right], \quad \mathbf{B}=\left[\begin{array}{cccc}
\mathbf{b}_{1} & \mathbf{0} & \cdots & \mathbf{0} \\
\mathbf{0} & \mathbf{b}_{2} & \ddots & \vdots \\
\vdots & \ddots & \ddots & \mathbf{0} \\
\mathbf{0} & \cdots & \mathbf{0} & \mathbf{b}_{N}
\end{array}\right]
$$

where $\mathbf{x}(t)$ is the state vector consisting of all generator states and $\mathbf{u}(t)$ contains all control signals. The matrices $\mathbf{A}, \mathbf{B}, \mathbf{A}_{i, j}, \mathbf{b}_{i}$ with $i, j \in\{1 \ldots N\}$ are of appropriate dimensions. The analysis in the next section is also applicable for a general large scale dynamical system with interconnections.

\section{PROBLEM FORMULATION}

In this section we design controllers based on standard LQR. Assume that the system in (2) is controllable with initial condition $\mathbf{x}(0)=\mathbf{x}_{0}$ in a regulation problem, and the states are directly available to the controller. We associate a quadratic performance index

$$
J=\int_{0}^{\infty}\left(\mathbf{x}^{T}(t) \mathbf{Q}_{\mathbf{x}} \mathbf{x}(t)+\mathbf{u}^{T}(t) \mathbf{Q}_{\mathbf{u}} \mathbf{u}(t)\right) d t
$$

where $\mathbf{Q}_{\mathbf{x}} \geq \mathbf{0}$ and $\mathbf{Q}_{\mathbf{u}}>\mathbf{0}$ are symmetric weighting matrices of appropriate dimensions.

\subsection{Networked Cooperative LQR}

First, we consider the cooperative case where an ideal communication network exists in the DPG such that the controller of each subsystem (generator) is able to receive state information from the others. In order to know the best possible performance as a baseline for further comparison, here the communication topology is assumed to be fully connected i.e. each generator can receive information from all other generators in the grid. A similar approach can be extended for non-fully connected communication topology.

The optimal control law for the system in (2) that minimizes the cost in (3) is given by

$$
\mathbf{u}_{\mathrm{SI}}(t)=-\mathbf{L}_{\mathrm{SI}} \mathbf{x}(t)
$$

where the state feedback gain $\mathbf{L}_{\mathrm{SI}}$ is

$$
\mathbf{L}_{\mathrm{SI}}=\mathbf{Q}_{\mathbf{u}}^{-1} \mathbf{B}^{T} \mathbf{S}_{\mathrm{SI}}
$$

and $\mathbf{S}_{\mathrm{SI}}$ is the solution of the algebraic Riccati equation

$$
\mathbf{A}^{T} \mathbf{S}_{\mathrm{SI}}+\mathbf{S}_{\mathrm{SI}} \mathbf{A}-\mathbf{S}_{\mathrm{SI}} \mathbf{B} \mathbf{Q}_{\mathbf{u}}^{-1} \mathbf{B}^{T} \mathbf{S}_{\mathrm{SI}}+\mathbf{Q}_{\mathbf{x}}=\mathbf{0} .
$$

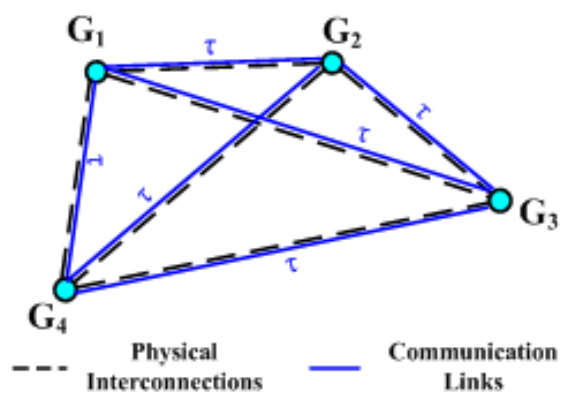

Fig. 2. Graph of a DPG with 4 generators $G_{1} \cdots G_{4}$ connected physically and by communication links with a constant time delay $\tau$.

Due to the LTI system with interconnections in (2), the state feedback gain matrix $\mathbf{L}_{\mathrm{SI}}$ can be formulated as

$$
\mathbf{L}_{\mathrm{SI}}=\left[\begin{array}{cccc}
\mathbf{l}_{1,1} & \mathbf{l}_{1,2} & \cdots & \mathbf{l}_{1, N} \\
\mathbf{l}_{2,1} & \mathbf{l}_{2,2} & \ddots & \vdots \\
\vdots & \ddots & \ddots & \mathbf{l}_{N-1, N} \\
\mathbf{l}_{N, 1} & \cdots & \mathbf{l}_{N, N-1} & \mathbf{l}_{N, N}
\end{array}\right]
$$

The non-diagonal terms $\mathbf{l}_{i, j}$ of appropriate dimension with $i, j \in\{1 \ldots N\}$ and $i \neq j$ in (5) show the state information exchange from other generators via the communication network for the computation of the local control signal.

Therefore, the local control law for a single generator $G_{i}$ can be written as

$$
\mathbf{u}_{i, \mathrm{SI}}(t)=-\sum_{j=1}^{N} \mathbf{l}_{i, j} \mathbf{x}_{j}(t) .
$$

With ideal communication, i.e. zero time delay, the optimal solution achieves the minimum cost

$$
J_{\mathrm{SI}}=\mathbf{x}_{0}^{T} \mathbf{S}_{\mathrm{SI}} \mathbf{x}_{0} \text {. }
$$

Now, let us consider the networked cooperative control with constant and identical time delay for all communication links. The graph of the power grid with physical and communication interconnections with time delay $\tau$ is illustrated in Figure 2, where the nodes represent the generators. From (6), the networked cooperative local controller for a single generator $G_{i}$ affected by time delay $\tau$ turns out to be

$$
\mathbf{u}_{i, \tau}(t)=-\mathbf{l}_{i, i} \mathbf{x}_{i}(t)-\sum_{j=1, j \neq i}^{N} \mathbf{l}_{i, j} \mathbf{x}_{j}(t-\tau) .
$$

Thus, the networked cooperative control with time delay for the overall system can be written by

$$
\mathbf{u}_{\tau}(t)=-\mathbf{L}_{\text {diag }} \mathbf{x}(t)-\mathbf{L}_{c r} \mathbf{x}(t-\tau),
$$

where the gain matrix $\mathbf{L}_{\text {diag }}$ and $\mathbf{L}_{c r}$ correspond to

$\mathbf{L}_{\text {diag }}=\left[\begin{array}{cccc}\mathbf{l}_{1,1} & \mathbf{0} & \cdots & \mathbf{0} \\ \mathbf{0} & \mathbf{l}_{2,2} & \ddots & \vdots \\ \vdots & \ddots & \ddots & \mathbf{0} \\ \mathbf{0} & \cdots & \mathbf{0} & \mathbf{l}_{N, N}\end{array}\right], \mathbf{L}_{c r}=\left[\begin{array}{cccc}\mathbf{0} & \mathbf{l}_{1,2} & \cdots & \mathbf{l}_{1, N} \\ \mathbf{l}_{2,1} & \mathbf{0} & \ddots & \vdots \\ \vdots & \ddots & \ddots & \mathbf{l}_{N-1, N} \\ \mathbf{l}_{N, 1} & \cdots & \mathbf{l}_{N, N-1} & \mathbf{0}\end{array}\right]$,

and the matrices $\mathbf{L}_{\text {diag }}, \mathbf{L}_{c r}$ and $\mathbf{L}_{\mathrm{SI}}$ satisfy the relation

$$
\mathbf{L}_{\mathrm{SI}}=\mathbf{L}_{\text {diag }}+\mathbf{L}_{c r} \text {. }
$$

In (8), the term $-\mathbf{L}_{\text {diag }} \mathbf{x}(t)$ implies the control actions based on the own states of each generator, while the term 
$-\mathbf{L}_{c r} \mathbf{x}(t-\tau)$ represents the control actions driven by the delayed states transmitted from other generators.

The closed loop dynamics with time delay is then given by

$$
\dot{\mathbf{x}}(t)=\mathbf{A}_{0} \mathbf{x}(t)+\mathbf{A}_{1} \mathbf{x}(t-\tau),
$$

with $\mathbf{A}_{0}=\left(\mathbf{A}-\mathbf{B L}_{\text {diag }}\right)$ and $\mathbf{A}_{1}=-\mathbf{B L}_{c r}$. The initial condition for the delayed states is defined as $\mathbf{x}(t-\tau)=\mathbf{x}_{0}$ for $0 \leq t<\tau$.

In a next step we determine the cost resulting from the networked cooperative LQR with delayed states information exchange. Recall the minimum cost $J_{\mathrm{SI}}$ achieved by the networked cooperative LQR with ideal communication defined in (7). Since the $\mathbf{S}_{\mathrm{SI}}$ is the solution of the algebraic Riccati equation in (4), the cost $J$ in (3) for a regulation problem with the initial state $\mathbf{x}_{0}$ can be formulated as

$$
\begin{aligned}
J= & \mathbf{x}_{0}^{T} \mathbf{S}_{\mathrm{SI}} \mathbf{x}_{0}+\int_{0}^{\infty}\left(\mathbf{u}(t)+\mathbf{Q}_{\mathbf{u}}^{-1} \mathbf{B}^{T} \mathbf{S}_{\mathrm{SI}} \mathbf{x}(t)\right)^{T} \mathbf{Q}_{\mathbf{u}} . \\
& \left(\mathbf{u}(t)+\mathbf{Q}_{\mathbf{u}}^{-1} \mathbf{B}^{T} \mathbf{S}_{\mathrm{SI}} \mathbf{x}(t)\right) d t \\
= & J_{\mathrm{SI}}+\int_{0}^{\infty}\left(\mathbf{u}(t)+\mathbf{L}_{\mathrm{SI}} \mathbf{x}(t)\right)^{T} \mathbf{Q}_{\mathbf{u}}\left(\mathbf{u}(t)+\mathbf{L}_{\mathrm{SI}} \mathbf{x}(t)\right) d t
\end{aligned}
$$

for any admissible $\mathbf{u}(t)$ (Kwong [2008]). Substituting $\mathbf{u}(t)$ in (11) by $\mathbf{u}_{\tau}(t)$ in (8) and using (9) we obtain

$$
\begin{aligned}
J_{\tau}= & J_{\mathrm{SI}}+\int_{0}^{\infty}\left(-\mathbf{L}_{\text {diag }} \mathbf{x}(t)-\mathbf{L}_{c r} \mathbf{x}(t-\tau)+\mathbf{L}_{\mathrm{SI}} \mathbf{x}(t)\right)^{T} . \\
& \mathbf{Q}_{\mathbf{u}}\left(-\mathbf{L}_{\text {diag }} \mathbf{x}(t)-\mathbf{L}_{c r} \mathbf{x}(t-\tau)+\mathbf{L}_{\mathrm{SI}} \mathbf{x}(t)\right) d t \\
= & J_{\mathrm{SI}}+\int_{0}^{\infty}\left[\mathbf{L}_{c r} \Delta \mathbf{x}_{\tau}(t)\right]^{T} \mathbf{Q}_{\mathbf{u}} \cdot\left[\mathbf{L}_{c r} \Delta \mathbf{x}_{\tau}(t)\right] d t
\end{aligned}
$$

and the increased amount $\Delta J_{\tau}$ of the cost $J_{\tau}$ relative to $J_{\mathrm{SI}}$ is given by

$$
\Delta J_{\tau}=\int_{0}^{\infty}\left[\mathbf{L}_{c r} \Delta \mathbf{x}_{\tau}(t)\right]^{T} \mathbf{Q}_{\mathbf{u}} \cdot\left[\mathbf{L}_{c r} \Delta \mathbf{x}_{\tau}(t)\right] d t \geq 0
$$

where $\Delta \mathbf{x}_{\tau}(t)=\mathbf{x}(t)-\mathbf{x}(t-\tau)$. The cost increase $\Delta J_{\tau}$ shows the performance degradation caused by time delay.

\subsection{LQR without Information Exchange}

Now, we assume there is no communication between the generators. In this case, a local controller for a single generator $G_{i}$ is defined as

$$
\mathbf{u}_{i, \mathrm{NSI}}(t)=-\mathbf{l}_{i, \mathrm{NSI}} \mathbf{x}_{i}(t),
$$

with $\mathbf{l}_{i, \text { NSI }}$ of appropriate dimension independent of the states of other generators.

The control law for the whole grid then becomes

$$
\mathbf{u}_{\mathrm{NSI}}(t)=-\mathbf{L}_{\mathrm{NSI}} \mathbf{x}(t),
$$

with a block diagonal state feedback gain matrix $\mathbf{L}_{\mathrm{NSI}}$

$$
\mathbf{L}_{\mathrm{NSI}}=\left[\begin{array}{cccc}
\mathbf{l}_{1, \mathrm{NSI}} & \mathbf{0} & \cdots & \mathbf{0} \\
\mathbf{0} & \mathbf{l}_{2, \mathrm{NSI}} & \ddots & \vdots \\
\vdots & \ddots & \ddots & \mathbf{0} \\
\mathbf{0} & \cdots & \mathbf{0} & \mathbf{l}_{N, \mathrm{NSI}}
\end{array}\right]
$$

is sought. The feedback gain matrix $\mathbf{L}_{\text {NSI }}$ with a block diagonal structure is computed by restricting $\mathbf{S}_{\mathbf{N S I}}$ to a positive definite, symmetric and block diagonal matrix

$$
\mathbf{L}_{\mathrm{NSI}}=\mathbf{Q}_{\mathbf{u}}^{-1} \mathbf{B}^{T} \mathbf{S}_{\mathrm{NSI}}
$$

with condition that $\mathbf{Q}_{\mathbf{u}}$ and $\mathbf{B}$ have block diagonal structure. Note that the resulting controller is suboptimal and the corresponding cost achieved by such controller with the initial condition $\mathbf{x}(0)=\mathbf{x}_{0}$ is given by Langholz [1979]

$$
J_{\mathrm{NSI}}=\mathbf{x}_{0}^{T} \boldsymbol{\Lambda} \mathbf{x}_{0}
$$

where $\boldsymbol{\Lambda} \in \Re^{2 N \times 2 N}$ is the positive definite and symmetric solution of the linear algebraic equation

$$
\begin{aligned}
\left(\mathbf{A}-\mathbf{B L}_{\mathrm{NSI}}\right)^{T} & \boldsymbol{\Lambda}+\boldsymbol{\Lambda}\left(\mathbf{A}-\mathbf{B L}_{\mathrm{NSI}}\right) \\
& +\mathbf{S}_{\mathrm{NSI}} \mathbf{B Q}_{\mathbf{u}}^{-1} \mathbf{B}^{T} \mathbf{S}_{\mathrm{NSI}}+\mathbf{Q}_{\mathbf{x}}=\mathbf{0} .
\end{aligned}
$$

\subsection{Benefit of Cooperative Control}

Notice that since LQR without information exchange is suboptimal, the cost $J_{\mathrm{NSI}}$ in (15) is larger than the cost $J_{\mathrm{SI}}$ of the networked cooperative LQR with ideal communication in (7). This means that a perfect cooperation between the generators is beneficial, i.e. results in a better performance.

On the other hand, time delay degrades the performance of the networked cooperative LQR as shown in (13). Therefore, one of the interesting questions is up to which time delay such a communication network is still beneficial for the overall system. This can be formulated as to find a maximal delay $\tau_{\max }$ defined as follows

Definition 1. The performance guaranteed time delay bound $\tau_{\max } \in[0, \infty)$ is defined as the maximum time delay

$$
\begin{aligned}
& \max \tau \text { s.t. } \\
& J_{\tau}(\tau) \leq J_{\mathrm{NSI}} .
\end{aligned}
$$

The question is, how this delay bound can be computed in a systematic way. To avoid computation of the cost over an infinite horizon with time delay; here we propose to approximate this bound. Note that for the comparison of performances, it is assumed that there exists $\mathbf{L}_{\mathbf{N S I}}$ that stabilizes the system.

\section{LMI APPROACH FOR PERFORMANCE GUARANTEED TIME DELAY BOUND APPROXIMATION}

The goal is to find a performance guaranteed time delay bound such that the the cost of the networked cooperative LQR is smaller than the cost of LQR without information exchange. As a first step, we propose to approximate this bound.

\subsection{Time Delay System Approximation}

In this section we introduce an LMI problem based on the first order Pade approximation for the time delay to approximately determine the performance guaranteed time delay bound.

The Laplace transform of the delayed state vector $\mathbf{x}(t-\tau)$ is known as $e^{-\tau s} \mathbf{X}(s)$, where $\mathbf{X}(s)$ is the Laplace transform of the undelayed state vector $\mathbf{x}(t)$. By using the first order Pade approximation we introduce a new state vector $\gamma(t)$ whose Laplace transform $\boldsymbol{\Gamma}(s)$ is defined by

$$
\boldsymbol{\Gamma}(s)=\frac{1-\frac{\tau}{2} s}{1+\frac{\tau}{2} s} \mathbf{X}(s) .
$$

Thus, $\gamma(t)$ in time domain represents an approximation for the delayed state vector $\mathbf{x}(t-\tau)$. Substituting the delayed 
state vector $\mathbf{x}(t-\tau)$ in (10) by the approximation $\gamma(t)$, the closed loop system with approximated time delay becomes

$$
\dot{\mathbf{x}}(t)=\mathbf{A}_{0} \mathbf{x}(t)+\mathbf{A}_{1} \gamma(t) .
$$

Next, transforming $\boldsymbol{\Gamma}(s)$ back to time domain and substituting $\dot{\mathbf{x}}(t)$ by (18), we obtain the dynamic of $\gamma(t)$ as

$$
\dot{\gamma}(t)=\left(-\frac{2}{\tau} \mathbf{I}-\mathbf{A}_{1}\right) \boldsymbol{\gamma}(t)+\left(\frac{2}{\tau} \mathbf{I}-\mathbf{A}_{0}\right) \mathbf{x}(t),
$$

with the identity matrix $\mathbf{I}$. As the initial condition for the approximated delayed state vector we set $\gamma(0)=\mathbf{x}_{0}$.

Combining (18) and (19), we define a new state vector

$$
\chi(t)=\left[\begin{array}{l}
\mathbf{x}(t) \\
\gamma(t)
\end{array}\right] \in \Re^{4 N \times 1}, \quad \chi(0)=\chi_{0}
$$

with the closed loop dynamic

$$
\dot{\chi}(t)=\tilde{\mathbf{A}} \chi(t),
$$

where

$$
\tilde{\mathbf{A}}=\left[\begin{array}{cc}
\mathbf{A}_{0} & \mathbf{A}_{1} \\
\frac{2}{\tau} \mathbf{I}-\mathbf{A}_{0} & -\frac{2}{\tau} \mathbf{I}-\mathbf{A}_{1}
\end{array}\right] .
$$

Consider the approximated delayed state $\gamma(t)$, the cost of networked cooperative LQR affected by the approximated time delay is given as

$$
\begin{aligned}
J_{\text {approx }}= & \mathbf{x}_{0}^{T} \mathbf{S}_{\mathrm{SI}} \mathbf{x}_{0}+\int_{0}^{\infty}\left[\mathbf{L}_{c r}(\mathbf{x}(t)-\gamma(t))\right]^{T} \mathbf{Q}_{\mathbf{u}} . \\
& {\left[\mathbf{L}_{c r}(\mathbf{x}(t)-\gamma(t))\right] d t }
\end{aligned}
$$

and by using $(20)$ the corresponding cost increase $\Delta J_{\text {approx }}$ relative to the minimum cost $J_{\mathrm{SI}}$ turns out to be

$$
\begin{aligned}
\Delta J_{\text {approx }} & =\int_{0}^{\infty}(\gamma(t)-\mathbf{x}(t))^{T} \mathbf{W}(\gamma(t)-\mathbf{x}(t)) d t \\
& =\int_{0}^{\infty} \chi^{T}(t) \mathbf{M} \chi(t) d t
\end{aligned}
$$

with

$$
\mathbf{W}=\mathbf{L}_{c r}^{T} \mathbf{Q}_{\mathbf{u}} \mathbf{L}_{c r}, \quad \mathbf{M}=\left[\begin{array}{c}
\mathbf{I} \\
-\mathbf{I}
\end{array}\right] \cdot \mathbf{W} \cdot[\mathbf{I}-\mathbf{I}] .
$$

Since $\mathbf{Q}_{\mathbf{u}}$ is positive definite, $\mathbf{W}$ and $\mathbf{M}$ are positive definite as well.

\subsection{LMI Approach}

Based on the closed loop system in $(21)$ and $\Delta J_{\text {approx }}$ in (22), an LMI approach is introduced to determine a time delay bound $\hat{\tau}_{\max }$ such that the following inequality

$$
\Delta J_{\text {approx }} \leq J_{\mathrm{NSI}}-J_{\mathrm{SI}}
$$

holds. The $\hat{\tau}_{\max }$ can be interpreted as an approximation of the performance guaranteed time delay bound $\tau_{\max }$ defined in section 3.3. Moreover, intensive numerical simulations indicate that the $\hat{\tau}_{\max }$ indeed is an upper bound to $\tau_{\max }$, since $\hat{\tau}_{\max }$ is larger than $\tau_{\max }\left(J_{\text {approx }} \leq J_{\tau}\right)$. The mathematical proof for this statement is still under investigation.

The value of $\hat{\tau}_{\max }$ is determined by a search algorithm where the delay is continuously increased while checking the conditions of the following theorem. First we define a Lyapunov function $V(\boldsymbol{\chi}(t))=\chi^{T}(t) \boldsymbol{\Xi} \chi(t)$.
Theorem 2. If there exists a positive definite matrix $\boldsymbol{\Xi}$ such that the following conditions hold

$$
\begin{aligned}
& \text { - } \boldsymbol{\Xi}>0 \\
& \text { - } \tilde{\mathbf{A}}^{T} \boldsymbol{\Xi}+\boldsymbol{\Xi} \tilde{\mathbf{A}}<0 \\
& \text { - } \boldsymbol{\Theta} \leq 0
\end{aligned}
$$

with $\boldsymbol{\Theta}=\mathbf{M}+\tilde{\mathbf{A}}^{T} \boldsymbol{\Xi}+\boldsymbol{\Xi} \tilde{\mathbf{A}}$ and the system (21) is stable. In addition, if $V(\chi(0)) \leq J_{\mathrm{NSI}}-J_{\mathrm{SI}}$ holds, then $\Delta J_{\text {approx }} \leq J_{\mathrm{NSI}}-J_{\mathrm{SI}}$.

Proof. By means of (21), the derivative of the Lyapunov function $V(\chi(t))$ is given by

$$
\begin{aligned}
\dot{V}(\boldsymbol{\chi}(t)) & =\dot{\boldsymbol{\chi}}(t)^{T} \boldsymbol{\Xi} \boldsymbol{\chi}(t)+\boldsymbol{\chi}(t)^{T} \boldsymbol{\Xi} \dot{\boldsymbol{\chi}}(t) \\
& =\boldsymbol{\chi}^{T}(t)\left(\tilde{\mathbf{A}}^{T} \boldsymbol{\Xi}+\boldsymbol{\Xi} \tilde{\mathbf{A}}\right) \boldsymbol{\chi}(t) .
\end{aligned}
$$

If $\tilde{\mathbf{A}}^{T} \boldsymbol{\Xi}+\boldsymbol{\Xi} \tilde{\mathbf{A}}<0$ i.e. $\dot{V}(\boldsymbol{\chi}(t))<0$, the closed loop system (21) is asymptotically stable.

Next we modify $\Delta J_{\text {approx }}$ in $(22)$ according to

$$
\begin{aligned}
\Delta J_{\text {approx }} & =\int_{0}^{\infty}\left(\boldsymbol{\chi}^{T}(t) \mathbf{M} \boldsymbol{\chi}(t)+\dot{V}(\boldsymbol{\chi})(t)\right) d t-\int_{0}^{\infty} \dot{V}(\boldsymbol{\chi}(t)) d t \\
& =\int_{0}^{\infty} \boldsymbol{\chi}^{T}(t)\left(\mathbf{M}+\tilde{\mathbf{A}}^{T} \boldsymbol{\Xi}+\boldsymbol{\Xi} \tilde{\mathbf{A}}\right) \boldsymbol{\chi}(t) d t-V(\infty)+V(0),
\end{aligned}
$$

where $V(\chi(\infty))=0$ and $V(\chi(0))=\chi_{0}^{T} \boldsymbol{\Xi} \chi_{0}$.

Furthermore, defining a matrix $\boldsymbol{\Theta}=\mathbf{M}+\tilde{\mathbf{A}}^{T} \boldsymbol{\Xi}+\boldsymbol{\Xi} \tilde{\mathbf{A}}$, if $\boldsymbol{\Theta}$ is negative semidefinite, then

$$
\Delta J_{\text {approx }}=\int_{0}^{\infty} \chi^{T}(t) \boldsymbol{\Theta} \chi(t) d t+V(\chi(0)) \leq V(\chi(0)) .
$$

Finally if $V(\chi(0)) \leq J_{\mathrm{NSI}}-J_{\mathrm{SI}}$, then

$$
\Delta J_{\text {approx }} \leq J_{\mathrm{NSI}}-J_{\mathrm{SI}} \text {. }
$$

By verifying the feasibility of the LMI problem in Theorem 2, the approximated performance guaranteed time delay bound $\hat{\tau}_{\max }$ can be determined in a systematic way. Alternatively, the LQ cost over infinite horizon in (3) would need to be evaluated in an approximate way.

\section{NUMERICAL EVALUATION}

As an example we consider a microgrid consisting of 5 generators with topology shown in Figure 1. The generators' parameters and the resistances of transmissions lines are chosen to be realistic as shown in Table 1 . The constant load $R$ is chosen to be $100 \Omega$. The weighting matrices in cost function (3) are $\mathbf{Q}_{\mathbf{x}}=\mathbf{I}$ and $\mathbf{Q}_{\mathbf{u}}=0.1 \cdot \mathbf{I}$, where $\mathbf{I}$ is the identity matrix.

Table 1. Simulation Parameters

\begin{tabular}{c|c|c|c|c}
\hline$G_{i}$ & $R_{i}[\Omega]$ & $k_{i}[\mathrm{Vs} / \mathrm{rad}]$ & $J_{i}\left[\mathrm{kgm}^{2} / \mathrm{s}^{2}\right]$ & $\beta_{i}[\mathrm{~s}]$ \\
\hline$G_{1}$ & 9 & 8.1942 & 1 & 4.0312 \\
$G_{2}$ & 6 & 4.5923 & 0.5037 & 7.6671 \\
$G_{3}$ & 7 & 9.9249 & 0.8929 & 3.2975 \\
$G_{4}$ & 5 & 3.5350 & 0.5934 & 2.2140 \\
$G_{5}$ & 11 & 11.7269 & 0.2071 & 1.4856 \\
\hline
\end{tabular}

The initial condition of the system is randomly chosen as 


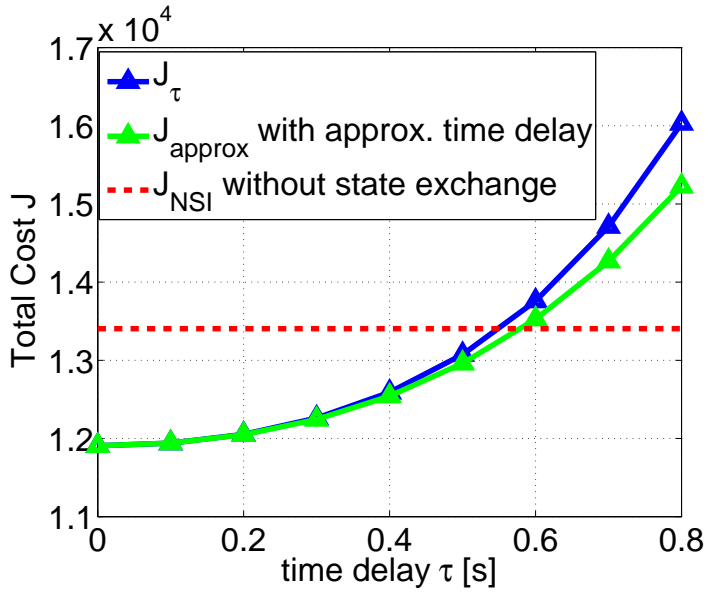

Fig. 3. Control performance in the presence of time delay.

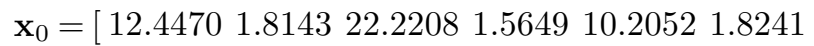

$$
\begin{aligned}
& \left.\begin{array}{llll}
28.4662 & 0.4444 & 8.6477 & 1.5048
\end{array}\right]^{T} .
\end{aligned}
$$

First, we simulate the dynamics of the DPG in MATLAB/SIMULINK over a time interval of $\mathrm{T}=70 \mathrm{~s}$. The time delay $\tau$ is increased from $0 s$ to $0.8 s$ in $0.1 s$ step. By analyzing the system poles, the simulated system turns to be stable for all considered time delay.

As shown in Figure 3, the system performance with networked cooperative LQR with no time delay $(\tau=0)$ is better than LQR without information exchange (red dashed line) indicated by a lower cost for the former one. This confirms that introducing communication network could be beneficial for the overall control performance. However, the cost $J_{\tau}$ of the networked cooperative LQR with time delay (blue line) rises with the increase of time delay. Beyond a certain delay called performance guaranteed time delay bound, the cost for networked cooperative controllers becomes larger than LQR without information exchange i.e. it is no more beneficial to exchange states information. By using a line search algorithm of comparison of the resulted LQ cost, the performance guaranteed time delay bound $\tau_{\max }$ is equal to $0.536 \mathrm{~s}$.

Next, we simulate the dynamics of DPG again with approximated time delay. As shown in Figure 3, the cost $J_{\text {approx }}$ (green line) also shows similar behavior with the increase of time delay. Then, the approximated performance guaranteed time delay bound $\left(\hat{\tau}_{\max }\right)$ for system with approximated delay is determined by evaluating the feasibility of the LMI in Theorem 2 using the YALMIP toolbox (Löfberg [2004]) with SEDUMI and PENBMI solver which are $\hat{\tau}_{\max }=0.560 \mathrm{~s}$ and $\hat{\tau}_{\max }=0.559 \mathrm{~s}$ respectively.

Thus, the simulation strengthens the indication that the approximated performance guaranteed time delay bound $\hat{\tau}_{\max }$ can also be interpreted as an upper bound of the true time delay bound. Naturally, we expect this value to increase, if the time delay is included in the design.

\section{CONCLUSION}

In this paper, the effect of time delay on the performance of a Distributed Power Grid with networked cooperative controllers is studied. Introducing a communication network results in a better performance, i.e. lower cost compared with controllers with only local information. However, in the presence of time delay, the cost is shown to be increased with the increase of time delay and beyond a certain point, the cost becomes larger than for controller without information exchange. Based on first order Pade approximation and by solving the LMI problem, the approximated performance guaranteed time delay bound is determined analytically. Future work includes more sophisticated control design (e.g. Hassan et al. [2007]) and the inclusion of other communication topologies and network effects.

\section{ACKNOWLEDGEMENTS}

The authors would like to thank Prof. Anuradha Annaswamy of MIT and Harald Voit of TUM for the several stimulating discussions on this topic.

\section{REFERENCES}

Bakule, L. (2008). Decentralized control: An Overview. Annual Reviews in Control, 32, 87-98.

Hassan, M. F., and Boukas, K. (2007). Multilevel Technique for Large Scale LQR with Time Delays and Systems Contraints. International Journal of Innovative Computing, Information and Control, 3(2), 419-434.

Zhang, W., Branicky, M. S., and Phillips, S. M. (2001). Stability of Networked Control Systems. IEEE Control Systems Magazine, 84-99.

Kwong, R. H. (2008). Lecture Notes - Control Systems, Chapter 6, Linear Quadratic Optimal Control. University of Toronto.

Langholz, G. (1979). A new lower bound on the cost of optimal regulators. IEEE Transactions on Automatic Control, 24(2), 353-354.

Löfberg, J. (2004). YALMIP : A toolbox for modeling and optimization in MATLAB. Proceedings of the CACSD Conference, 284-289.

Rotkowitz, M., and Lall, S. (2006). A Characterization of Convex Problems in Decentralized Control. IEEE Transactions on Automatic Control, 51(2), 1984-1996.

Liu, P. (2003). Exponential Stability for Linear TimeDelay Systems with Delay Dependence. Journal of the Franklin Institute, 340(6) 481-488.

Marwali, M. N., and Keyhani, A. (2004). Control of Distributed Generation Systems - Part I Voltages and Currents Control, IEEE Transcations on Industrial Electronics, 19(6), 1541-1550.

Venkat, A. N., Hiskens, I. A., Rawlings, J. B., and Wright, S. J. (2008). Distributed MPC Strategies With Application to Power System Automatic Generation Control, IEEE Transactions on Control Systems Technology, 16(6), 1192-1206.

Negenborn, R. R. (2007). Multi-Agent Model Predictive Control with Applications to Power Networks, Doctoral Dissertation, Technische Universiteit Delft.

Richard, J. -P. (2003). Time-delay Systems, An Overview of Some Recent Advances and Open Problems, Automatica, 39(10), 1667-1694. 